\title{
STIMULUS-SPECIFIC MECHANISMS OF VISUAL SHORT-TERM MEMORY
}

\author{
Svein Magnussen, ${ }^{1}$ Mark W. Greenlee, ${ }^{2}$ Rolf Asplund ${ }^{1}$ and Stein Dyrnes ${ }^{1}$

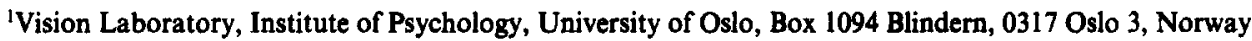 \\ and 'Department of Neurophysiology, University of Freiburg, Germany
}

(Received 5 February 1990; in revised form 5 December 1990)

\begin{abstract}
The retention of spatial information in visual short-term memory was assessed by measuring spatial frequency discrimination thresholds with a two-interval forced-choice task varying the time interval between the two gratings to be compared. The memory of spatial frequency information was perfect across 10-sec interstimulus intervals. Presentation of a "memory masker" grating during the interstimulus interval may interfere with short-term memory. This interference depends on the relative spatial frequency of the test and masker gratings, with maximum interference at spatial frequency differences of 1-1.5 octaves and beyond. This range of interference with short-term memory is comparable to the bandwidth of sensory masking or adaptation. A change of the relative orientation of test and masker gratings does not produce interference with spatial frequency discrimination thresholds. These results suggest stimulus-specific interactions at higher-level representations of visual form.
\end{abstract}

Short-term memory Spatial frequency Stimulus specificity

\section{INTRODUCTION}

Neurophysiological studies of the visual system in the cat and monkey provide strong evidence that the initial coding of shape is performed by a number of parallel mechanisms or channels of the visual cortex, each responding to a restricted range of spatial frequencies (size) and orientations (Rose \& Dobson, 1985; Shapley \& Lennie, 1985; De Valois \& De Valois, 1988). The psychophysical techniques of masking and selective adaptation, which are used to infer the bandwidth of spatial channels in man (Olzak \& Thomas, 1986; De Valois \& De Valois, 1988) most likely operate on such low-level neural representations because the effects are conjointly selective to orientation and spatial frequency, and tied to local retinal coordinates (Blakemore, Garner \& Sweet, 1972). At higher levels of representation which form the basis of perceptual discrimination and identification, size and orientation are independently processed (Burbeck \& Regan, 1983; Bradley \& Skottun, 1984) and abstracted from the retinal coordinates (Burbeck, 1987).

There is a growing interest among vision scientists in the transfer of information from sensory analysis to memory and the retention of information about stimulus attributes such as spatial frequency, orientation and movement for which specific sensory channels have been demonstrated (Magnussen, Landrø \& Johnsen, 1985; Regan, 1985a; Harvey, 1986; Vogels \& Orban, 1986; Magnussen, Asplund, Dyrnes \& Greenlee, 1988; Obergfell, Greenlee \& Magnussen, 1989; Magnussen, Greenlee, Asplund \& Dyrnes, 1990). In two recent studies (Regan, 1985; Magnussen et al., 1990), the short-term memory for spatial frequency was assessed by a spatial-frequency discrimination task using a two-interval forced-choice procedure with a variable interstimulus interval between the two gratings to be discriminated. A successful discrimination depended upon comparing the spatial frequency of the second grating with the stored representation of the first grating; and the discrimination threshold $\Delta F / F$ is a measure of the fidelity of this storage.

The results of these experiments showed that the short-term retention of the spatial frequency of simple gratings was perfect for the interstimulus intervals tested (up to $30 \mathrm{sec}$, Magnussen et al., 1990), with discrimination thresholds around $4-5 \%$ for gratings of medium contrast and spatial frequencies in the range of 5-20 c/deg. The perceptual discrimination based on the memory is equally precise for gratings of parallel and orthogonal orientations (Regan, 1985a,b; Magnussen et al., 1990), suggesting that spatial discrimination and spatial memory 
are based on common higher-level representations. This is further supported by the fact that discrimination thresholds may be increased by reducing contrast to near threshold (Watson \& Robson, 1981) or shortening the exposure times, but the degraded information is perfectly stored (Magnussen et al., 1990). Storage must therefore occur central to sensory analysis, at or beyond the level of perceptual discrimination.

These findings were a little surprising because Harvey (1986) found normal short-term memory decay for complex gratings over a 10 -sec interstimulus interval. According to Harvey (1986) the short-term memory of the complex gratings was based on retention of the medium spatial frequencies. The results with simple gratings showed, however, no differences in the memory for spatial frequencies in the range of 0.7-20 c/deg (Magnussen et al., 1990), so the decay of complex stimuli cannot be explained simply by selective retention of certain spatial frequencies.

To account for the discrepant results with simple and complex gratings we tentatively suggested that visual spatial short-term memory might be organized in terms of a set of parallel stores along the spatial frequency spectrum. The decay of the memory trace for complex gratings, as found by Harvey (1986), might then be due to interference between stores during retention or during the read-out of the stored sensory information.

The present study provides further evidence for this analogy between sensory processing and the mechanism of visual spatial short-term memory, demonstrating frequency-specific interference with the short-term memory of simple gratings by a third, "memory masker" grating exposed during the inter-stimulus interval in the two-interval forced-choice (2IFC) task. Some of these results have been reported in abstract form (Magnussen et al., 1988).

\section{METHOD}

The scheme of these experiments is illustrated in Fig. 1. Sinewave luminance gratings were presented on a high-resolution cathode ray tube (Joyce Electronics, Cambridge, U.K.) with a green (P4) phosphor and a frame rate of $100 \mathrm{~Hz}$. The control voltages determining the spatial frequency, contrast, spatial position and temporal frequency of the gratings were under the control of a microprocessor. The display had a spaceaveraged luminance of $150 \mathrm{~cd} / \mathrm{m}^{2}$, which was masked down to a circular field having a diam- eter of $8 \mathrm{deg}$ visual angle at $114 \mathrm{~cm}$ viewing distance.

Spatial-frequency discrimination thresholds were measured in a 2 IFC procedure. Brief $(200$ $\mathrm{msec}$ ) exposure to a vertical sine-wave grating of defined spatial frequency, spatial phase and contrast was followed by a 10 -sec interstimulus interval (ISI) during which the observer viewed a blank screen. This was followed by the presentation of a second grating, which differed in spatial frequency by $\Delta f$. The reference spatial frequency $F_{1}$ and test spatial frequency $F_{2}=F_{1}$ $\pm \Delta f$, were randomly presented either in the first or second interval. The observer pressed one of two buttons to signal in which interval the higher spatial frequency was presented. Frequency discrimination thresholds were estimated using a staircase procedure that controlled the value of $\Delta f$, which could range from 1 to $20 \%$ in increments of $1 \%$ of the reference spatial frequency. A maximum likelihood algorithm (Best-Pest; Lieberman \& Pentland, 1982) was used to provide an efficient estimate of the threshold which was defined as the $75 \%$ correct performance level. This procedure assumes that the psychometric function has a normal sigmoidal shape. It can be described, for the 2IFC-procedure by the logit function:

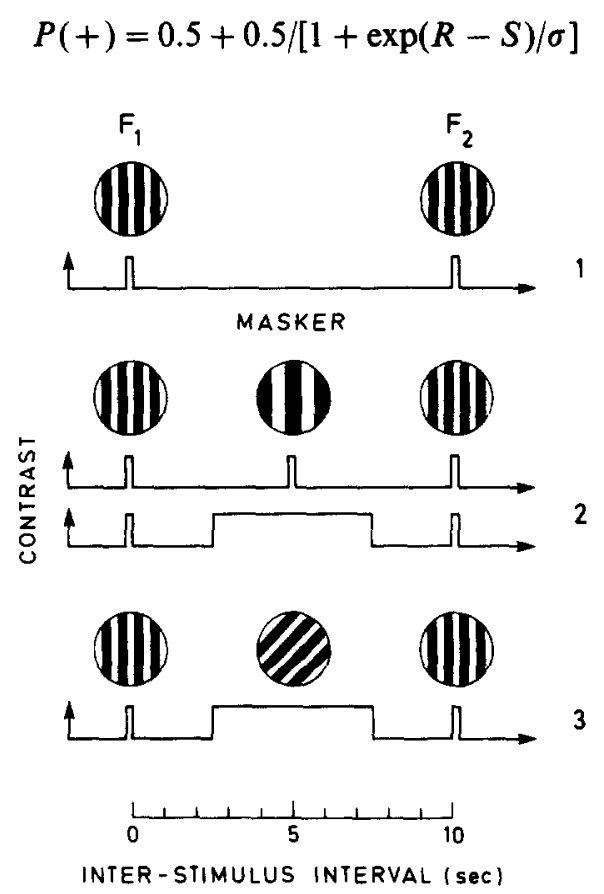

Fig. 1. Stimuli and time relations of the experiments. The interstimulus interval (ISI) between the luminance gratings to be discriminated $\left(F_{1}\right.$ and $\left.F_{2}\right)$ is filled with a blank screen (1) or a masker grating that differ in either spatial frequency (2) or orientation (3). Diagram 2 indicates the duration of the shortest and longest exposure of the masker grating. 
where $P(t)$ is the probability of correct response, $R$ is the total number of steps spanning the range of possible stimulus values, $S$ corresponds to each intensity step in the staircase and $\sigma$ is the standard deviation controlling the slope of the psychometric function. After each trial a likelihood function is calculated to determine at which step the difference threshold has the greatest likelihood of being located. The maximum likelihood estimation was determined after 30-40 trials. The data points shown in the figures are based on a minimum of three such measurements.

The reference spatial frequency was randomly varied from trial to trial by adding or subtracting a small random value (maximum $10 \%$ ) to the initial reference frequency. The contrast of the reference and test gratings was likewise randomly varied from presentation to presentation by adding or subtracting a small random value (maximum also $10 \%$ ) to the reference contrast. Spatial phase was randomly chosen to vary from 0 to 359 deg phase angle on a random basis. The random number generator was based on a even probability distribution with a mean of zero and a range that varied from -1 to 1 . Although a normal probability distribution would have assured that these random numbers would have added to zero over a given measurement, the even distribution used here provided numbers whose sum did not substantially differ from zero. These manipulations prevented the subjects from developing a long-term representation of the reference grating. It also made it impossible to use some form of magnitude estimation to code relative spatial frequencies, as the reference frequency was changing from trial to trial by a value $2 \times$ larger than the baseline difference threshold. The judgements had to be based solely on comparisons of the relative spatial frequency of (stored representation of) the first grating and second grating on the individual trials.

In the majority of the experiments to be presented, a third "memory masker" grating was displayed midway during the interstimulus interval (Fig. 1, diagrams 2 and 3). The contrast of the masker grating was $15 \%$ and the exposure duration was either $0.2,1.6$ or 5.0 sec in different experiments. Note that the on- and off-set of the masker were well outside the range of sensory masking on either the test or the reference grating for all mask durations (Breitmeyer, 1984). The observer was asked to scan the field during the exposure of the masker to avoid formation of afterimages which otherwise might have interfered with the frequency discrimination task. The relative spatial frequency or orientation of the masker grating was varied within a range of \pm 3 octaves and from 0 to $90 \mathrm{deg}$, respectively, from the initial reference spatial frequency or orientation in separate runs.

Authors RA, SD and MWG and one naive subject JO participated in the experiments. All had normal or corrected-to-normal vision.

\section{RESULTS}

We first repeated the short-term memory experiment without memory masking, serving as reference data for the subsequent experiments. Figure 2 shows the spatial frequency discrimination threshold $\Delta f / f$ for 1 and $10-\mathrm{sec}$ ISIs, for $5 \mathrm{c} / \mathrm{deg}$ reference frequency and vertical test and reference gratings. As already shown by previous experiments (Regan, 1985a; Magnussen et al., $1990)$ there is no short-term memory decay of spatial frequency information.

The scheme of the interference experiments, in which a "memory" masker grating was added during the ISI, is illustrated in diagrams 2 and 3 in Fig. 1. In the first series of experiments the test/reference and masker gratings had the same

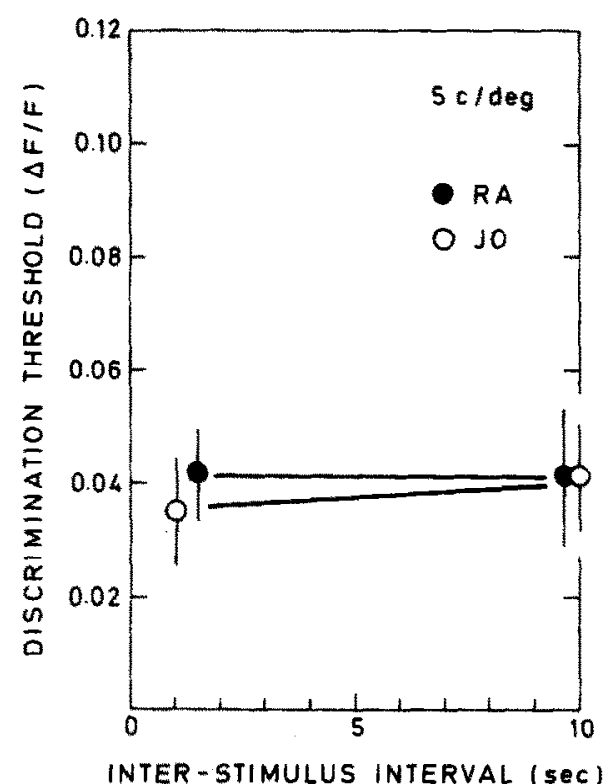

Fig. 2. The spatial frequency discrimination threshold for 1 . and 10-sec ISIs in the absence of a memory masker grating. The reference spatial frequency was $5.0 \mathrm{c} / \mathrm{deg}$ and grating contrast was $15 \%$. Data points represent the means of three measurements for subject JO and nine measurements for RA; vertical bars indicate \pm 1 SEM. 


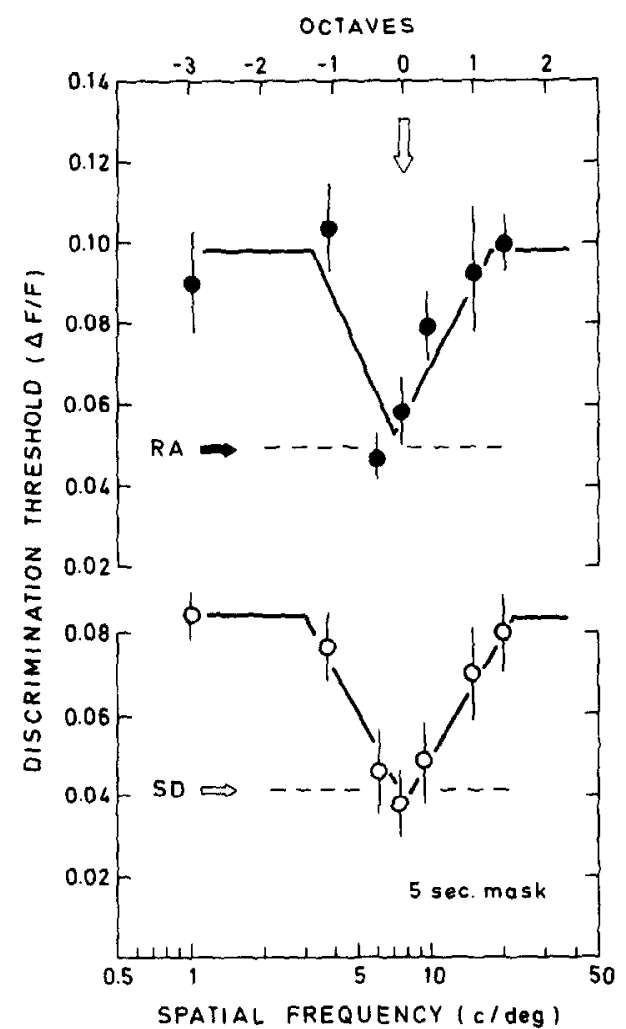

Fig. 3. The discrimination threshold for $7.5 \mathrm{c} / \mathrm{deg}$ reference spatial frequency (indicated by vertical arrow) as a function of the spatial frequency of the memory masker grating. The ISI was $10 \mathrm{sec}$ and duration of the masker was $5 \mathrm{sec}$. Results of two subjects, each data point is the mean of at least four measurements. Reference thresholds for spatial frequency discrimination with a blank interstimulus interval are indicated by dashed lines.

orientation, and the frequency of the masker was varied; within each experiment the various masker frequencies were tested in balanced order. Figures 3-5 show results for three different masker durations.

Figure 3 plots the discrimination threshold as a function of the frequency of the masker grating (lower abscissa) and the difference between reference frequency and masker frequency in octaves (upper abscissa), with a 5-sec masker duration and $7.5 \mathrm{c} / \mathrm{deg}$ reference spatial frequency (indicated by vertical arrow).

Contrary to intuitive expectation no change in discrimination threshold was found when the frequency of the masker matched reference spatial frequency, but progressively higher discrimination thresholds were obtained with increasing spatial frequency difference between test/reference and masker gratings. The threshold values level off at a frequency difference of approximately one octave in either direction, at which point the discrimination thresholds

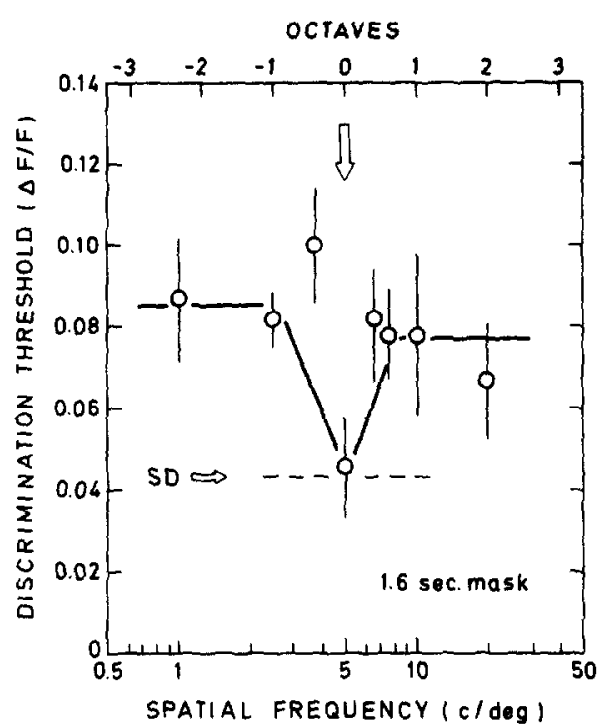

Fig. 4. Same as Fig. 3, but the reference spatial frequency was $5 \mathrm{c} / \mathrm{deg}$, and the duration of the masker was $1.6 \mathrm{sec}$.

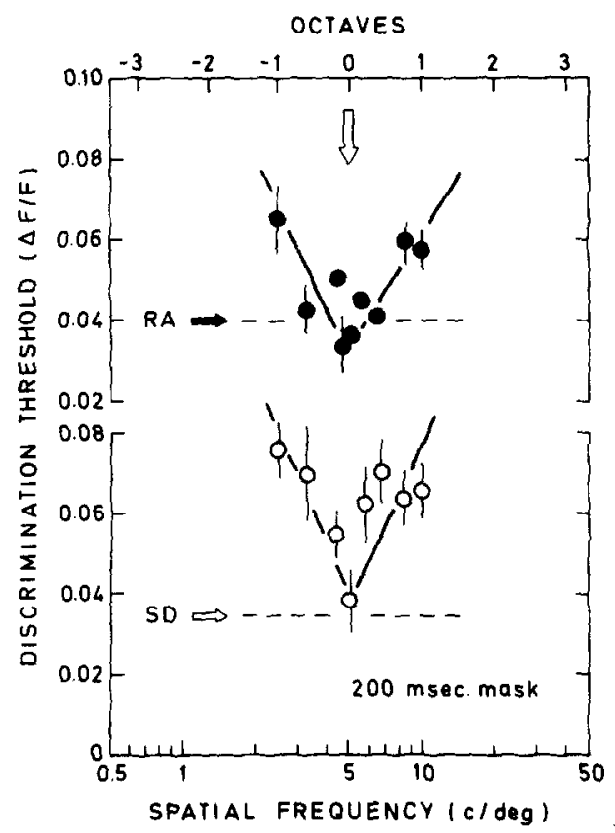

Fig. 5. Same as Fig. 3, but the reference spatial frequency was $5 \mathrm{c} / \mathrm{deg}$ and the duration of the masker was $0.2 \mathrm{sec}$. Each point is the mean of at least eight measurements.

are about twice the "unmasked" thresholds (indicated by horizontal dashed lines). The amount of threshold change and the range of masker frequencies over which memory masking is observed are quite consistent across the experiments and apparently do not depend on the duration of the masker. Figures 4 and 5 show the results of similar experiments for $5 \mathrm{c} / \mathrm{deg}$ reference frequency, and 1.6 and $0.2 \mathrm{sec}$ masker durations, respectively. There is no obvious effect 
of masker duration on the spatial frequency discrimination threshold.

The result with a brief masker is a very strong indication that the threshold elevation observed in our experiments is not produced by spatial adaptation. However, since adaptation has been reported to produce similar effects on spatial frequency discrimination thresholds (Regan \& Beverly, 1983), we specifically checked this point in the following control experiment: the contrast thresholds for grating detection were compared for a test grating of $5 \mathrm{c} / \mathrm{deg}$ presented alone and presented $2.5 \mathrm{sec}$ after a 5 -sec adapting grating of matched frequency, with adapting and test stimuli cycled as in the discrimination experiments. This control experiment is thus similar to the memory masking experiment with 5 -sec maskers. No contrast threshold elevation was observed with this adapt-test delay, in conformity with the results of independent experiments on the decay of threshold elevation for low contrast adapting gratings and short adapting times (Greenlee, Georgeson, Magnussen \& Harris, 1991).

In contrast to the above findings, the experiments with test/reference and masker gratings of matched spatial frequency but different orientation gave no indication of a memory masking effect on the spatial frequency discrimination thresholds. Figure 6 plots the spatial frequency discrimination threshold for vertical gratings of $5 \mathrm{c} / \mathrm{deg}$ reference spatial frequency as a function of the orientation of a $5 \mathrm{c} / \mathrm{deg}$ masker grating, and clearly shows that the short-term memory for spatial frequency is not disrupted by subsequent (irrelevant) information about orientation.

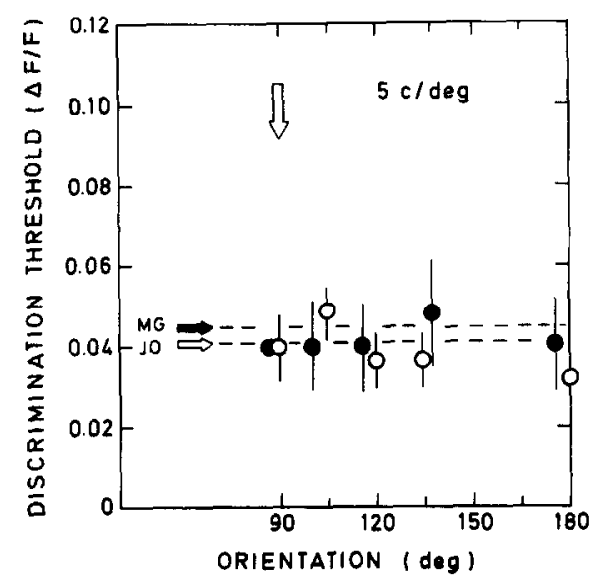

Fig. 6. Discrimination thresholds for vertical gratings of $5 \mathrm{c} / \mathrm{deg}$ reference spatial frequency as a function of the orientation of a $5 \mathrm{c} / \mathrm{deg}$ masker grating. Duration of the masker was $5 \mathrm{sec}$. Each point is the mean of three measurements.

\section{DISCUSSION}

The results of this study show that the shortterm memory for the spatial frequency of simple luminance gratings as measured by 2IFC determinations of the spatial frequency discrimination threshold, may be disrupted by presenting a masker grating during the retention interval. Masker gratings of either higher or lower frequencies than the reference spatial frequency raise the discrimination threshold. The range of interference compares with bandwidth estimates of spatial-frequency selective sensory channels obtained by conventional masking or adaptation regimes (Olzak \& Thomas, 1986). Furthermore, since both masking and adaptation may produce effects on spatial frequency discrimination thresholds that are analogous to "memory masking" the possibility that the present results could be explained in terms of such low-level sensory processes should be considered before discussing implications for visual short-term memory.

Regan (1985b) has shown that a masker grating presented simultaneously interferes with spatial frequency discrimination thresholds, but in the present experiments the on-set and off-set times of test and masker gratings were well outside the reach of masking effects (Breitmeyer, 1984). Explanations in terms of masking via afterimages are ruled out by the experiment with very brief, low-contrast maskers (Fig. 5).

For similar reasons it is unlikely that contrast adaptation would play a role. Regan and Beverly (1983) have reported that 5 min adaptation to high-contrast gratings produce effects similar to simultaneous masking, by increasing the spatial frequency discrimination thresholds for frequencies one octave away. However, we failed to find any adapting effect of the masker when testing contrast thresholds of the test gratings under conditions equivalent to the longest masker duration, and it is of course quite impossible that a $200 \mathrm{msec}$ masker would produce any noticeable adaptation with such delays (Greenlee et al., 1991).

Further evidence that the spatial-frequency dependent increase in the discrimination threshold is not due to factors operating on low-level sensory representations is found in the results of Fig. 6. The complete absence of a threshold increase for discrimination of vertical gratings at any orientation of a masker of matched frequency is only expected if the masker interferes at a level where orientation and spatial 
frequency are processed as separate attributes. In sensory adaptation and masking these attributes are linked (Olzak \& Thomas, 1986). Independence of spatial frequency and orientation has been demonstrated for the discrimination of both attributes (Burbeck \& Regan, 1983; Bradley \& Skottun, 1984), and for the short-term retention of spatial frequency (Regan, 1985a; Magnussen et al., 1991). Burbeck (1987) have shown that spatial-frequency discrimination is performed on basis of perceived spatial frequency independent of retinal spatial frequency, whereas spatial adaptation closely follows the retinal coordinates. It is likely that the type of interference produced by memory masking operates on higher-level representations on which the perceptual discrimination of form is based. Experiments on the short-term memory of naturalistic pictures have demonstrated "conceptual" masking with short delays between test and masker stimuli, but outside the range of sensory masking, and suggest that an analogous interference with visual short-term memory can take place at several levels of processing (Intraub, 1984; Lofthus \& Ginn, 1984).

From the literature on visual short-term memory (Humphreys \& Bruce, 1989) it is not surprising that distractor or masker stimuli interfere with short-term memory. The unexpected finding is the stimulus dependence of this interference, suggesting specific interactions rather than general distraction, enhanced uncertainty or overload of the memory mechanism. Such stimulus specific effects have been known for some time to exist in audition. Deutch and Feroe (1975) have shown that the short-term memory for pitch is disturbed by interpolating tones. They report that memory facilitation may occur when the distractor and test tones are similar, but that recognition errors rise as the pitch difference between the two tones is increased. As a possible explanation of such stimulus specific interactions Deutch and Feroe (1975) propose a model for the organization of pitch memory in terms of a memory array of stores linked in a lateral inhibitory network. We have in a previous paper (Magnussen et al., 1990) briefly proposed an analogous model for visual spatial short-term memory, in which the spatial frequency content of an image is represented in visual short-term memory in partial analogy to its sensory representation, by a set of parallel mechanisms coding a restricted range of frequencies. With such an arrangement the spatial-frequency specific interference might result from some kind of inhibitory interaction between the stores either during storage or in the read-out from the memory stores. There is psychophysical evidence for inhibition between spatial-frequency channels from selective adaptation experiments (Tolhurst \& Barfield, 1978; Greenlee \& Magnussen, 1988), and these interactions appear to have their analogues at several levels of visual processing.

The short-term memory decay in the absence of specific distractors reported for complex gratings (Harvey, 1986) are explained by this model in the same way as cognitive masking is explained, as arising from interactions between the individual frequency components. The decay in short-term memory for black-white block designs (Phillips, 1974, 1983; Inui, 1988) might be explained in a similar manner. However, the question of spatial frequency complexity and visual retention must be further examined.

There is recent neurophysiological evidence that pictorial short-term memory is represented by neural activity in cortical areas concerned with visual processing rather than in a specialized memory area from both single-unit studies on behaving monkeys (Miyashita \& Chang, 1988) and regional blood-flow studies on man (Roland, 1989). Such findings add credibility to the general hypothesis of a close connection between perceptual discrimination and the mechanism of visual short-term memory. Preliminary evidence from experiments on the short-term memory for motion (Obergfell et al., 1989) suggest that our findings for spatial frequency can be generalized to the memory representation of other basic stimulus attributes.

Acknowledgements - This study was supported by the grants from the Norwegian Research Council for Science and the Humanities and the Alexander von Humboldt-Stiftung (Germany) to SM. MWG was supported by Deutsche Forschungsgemeinschaft, SFB 325 (Germany). We thank L. G. Nilsson for comments on the manuscript.

\section{REFERENCES}

Blakemore, C., Garner, E. T. \& Sweet, J. A. (1972). The site of size constancy. Perception $1,111-119$.

Bradley, A. \& Skottun, B. C. (1984). The effect of large orientation and spatial frequency differences on spatial discriminations. Vision Research, 24, 1889-1896.

Breitmeyer, B. (1984). Visual masking. An integrative approach. Oxford: Oxford University Press.

Burbeck, C. A. (1987). Locus of spatial-frequency discrimination. Journal of the Optical Society of America A, 4, 1907-1813. 
Burbeck, C. A. \& Regan, D. (1983). Independence of orientation and size in spatial discrimination. Journal of the Optical Society of America, 73, 1691-1694.

Deutsch, D. \& Feroe, J. (1975). Disinhibition in pitch memory. Perception and Psychophysics, 17, 320-324.

De Valois, R. L. \& De Valois, K. K. (1988). Spatial vision. Oxford: Oxford University Press.

Greenlee, M. W. \& Magnussen, S. (1988). Interactions among spatial-frequency and orientation channels adapted concurrently. Vision Research, 28, 1303-1309.

Greenlee, M. W., Georgeson, M. A., Magnussen, S. \& Harris, J. P. (1991). The time course of adaptation to spatial contrast. Vision Research, 31, 223-246.

Harvey, L. O. Jr (1986). Visual memory: what is remembered? In Klix, F. \& Hagendorf, H. (Eds.), Human memory and cognitive capabilities, pp. 173-187. North-Holland Elsevier.

Humphreys, G. W. \& Bruce, V. (1989) Visual cognition. Computational, experimental and neuropsychological perspectives. London: Earlbaum.

Intraub, H. (1984). Conceptual masking: The effects of subsequent events on memory for pictures. Journal of Experimental Psychology: Learning, Memory and Cognition, 10, 115-125.

Inui, T. (1988). Properties of human visual memory for block patterns. Biological Cybernetics, 59, 179-187.

Liberman, H. R. \& Pentland, A. P. (1982). Microcomputerbased estimation of psychophysical thresholds: The best PEST. Behavioural Research Methods and Instrumentation, 14, 21-25.

Lofthus, G. R. \& Ginn, M. (1984). Perceptual and conceptual masking of pictures. Journal of Experimental Psychology: Learning, Memory and Cognition, 10, 435-441.

Magnussen, S., Landre, N. I. \& Johnsen, T. (1985). Visual half-field symmetry in orientation perception. Perception, 14, 265-273.

Magnussen, S., Asplund, R, Dyrnes, S. \& Greenlee, M. W. (1988). Spatial selectivity of the short-term memory image. Perception, 17, A54-55.

Magnussen, S., Greenlee, M. W., Asplund, R. \& Dyrnes, S. (1990). Perfect visual short-term memory for periodic patterns. European Journal of Cognitive Psychology, 2, 345-362.

Miyashita, Y. \& Chang, H. S. (1988). Neuronal correlate of pictorial short-term memory in the primate temporal cortex. Nature, London, 331, 68-70.

Obergfell, J., Greenlee, M. W. \& Magnussen, S. (1989). Short-term memory for motion: Temporal-frequency discrimination of drifting gratings as a function of interstimulus interval. Perception, 18, A38.

Olzak, L. A. \& Thomas, J. (1986). Seeing spatial patterns. In Boff, K., Kaufman, L. \& Thomas J. P. (Eds.), Handbook of perception and performance, Vol. I, Sensory processes and perception. New York: Wiley-Interscience.

Phillips, W. A. (1974). On the distinction between sensory storage and short-term visual memory. Perception and Psychophysics, 16, 283-290.

Phillips, W. A. (1983). Short-term visual memory. Philosophical Transactions of the Royal Society of London B, 302, 295-309.

Regan, D. (1985a). Storage of spatial-frequency information and spatial-frequency discrimination. Journal of the Optical Society of America, A, 2, 619-621.

Regan, D. (1985b). Masking of spatial-frequency discrimination. Journal of the Optical Society of America A, 2, 1153-1159.

Regan, D. \& Beverly, K. I. (1983). Spatial-frequency discrimination and detection: Comparison of postadaptation thresholds. Journal of the Optical Society of America, 73, $1684-1690$.

Roland, P. E. (1989). Paper presented at the ESF workshop on dynamic aspect of learning and memory, Zeist, The Netherlands, 1-3 December.

Rose, D. \& Dobson, V. G. (1985) Models of the visual cortex. New York: Wiley.

Shapley, R. \& Lennie, P. (1985). Spatial frequency analysis in the visual system. Annual Review of Neuroscience, 8 , $547-583$.

Tolhurst, D. J. and Barfield, L. P. (1978). Interactions between spatial frequency channels. Vision Research, 18 , 951-958.

Vogels, R. \& Orban, G. A. (1986). Decision processes in visual discrimination of line orientation. Joumal of Experimental Psychology: Human Perception and Performance, 12, 115-132.

Watson, A. B. \& Robson, J. G. (1981). Discrimination at threshold: Labelled detectors in human vision. Vision Research, 21, 1115-1121. 